\title{
Solid-liquid phase transition of Lennard-Jones fluid in slit pores under tensile condition
}

\section{$\operatorname{AUTHOR}(\mathrm{S}):$}

Miyahara, M; Kanda, H; Shibao, M; Higashitani, K

\section{CITATION:}

Miyahara, M ...[et al]. Solid-liquid phase transition of Lennard-Jones fluid in slit pores under tensile condition. JOURNAL OF CHEMICAL PHYSICS 2000, 112(22): 9909-9916

\section{ISSUE DATE:}

2000-06-08

URL:

http://hdl.handle.net/2433/39726

\section{RIGHT:}

Copyright 2000 American Institute of Physics. This article may be downloaded for personal use only. Any other use requires prior permission of the author and the American Institute of Physics. 


\title{
Solid-liquid phase transition of Lennard-Jones fluid in slit pores under tensile condition
}

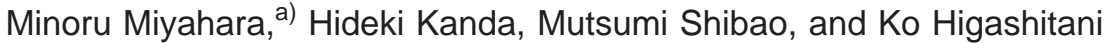 \\ Department of Chemical Engineering, Kyoto University, Kyoto 606-8501, Japan
}

(Received 10 December 1999; accepted 15 March 2000)

\begin{abstract}
The effect of equilibrium vapor-phase pressure onto freezing of a simple fluid in a nanopore is examined. We employ a molecular dynamics (MD) technique in a unit cell with imaginary gas phase, which has the benefit of easy determination of equilibrium vapor pressure. The method is shown to give consistent results with those by the grand canonical Monte Carlo (GCMC) method, and to have better feature of smaller degree of hysteresis between freezing and melting. The MD simulations showed liquid-solid phase transitions, at a constant temperature, with the variation in the equilibrium vapor-phase pressure below the saturated one. Thus-determined solid-liquid coexistence lines exhibited significant dependence of the freezing point against small changes in the bulk-phase vapor pressure, which implies the importance of tensile effect on freezing in nanopores. The capillary effect on the shift in freezing point was successfully described by a simple model based on continuum and isotropic assumption, even in a pore as small as $2 \mathrm{~nm}$ in width. (C) 2000 American Institute of Physics. [S0021-9606(00)70622-2]
\end{abstract}

\section{INTRODUCTION}

While capillary condensation in porous media is quantitatively well understood, only limited information is available for freezing/melting phenomena in nanoscale pores, even for simple systems. Understanding of solid-liquid transition in nanopores is of much importance in nanofabrications, nanotribology, and in characterization of pore sizes, as well as a basis for various operations employing nanoporous materials.

Many experimental studies of freezing in various porous solids have reported that freezing points are usually lowered. ${ }^{1-11}$ The materials used in the studies include Vycor glass, controlled pore glass, and various types of silica gels. Apparently however, variety is not sufficient to withdraw the overview of the phenomena: Most of the materials consist of silica or similar oxides, and their pores are roughly cylindrical, some of which cannot be well-characterized. Examinations of various systems, e.g., with various materials, other types of well-defined pore geometry, and with various condition in equilibrium bulk phase, are desired to find out the general trends of freezing in confined space, and its mechanisms.

While experimental measurements may suffer from combined effects of some factors that affect freezing phenomena, molecular simulation techniques are appropriate for examining them separately. Several molecular simulation studies have been carried out recently to clarify effects of some important factors. Miyahara and Gubbins ${ }^{12}$ used the grand canonical Monte Carlo (GCMC) simulation to observe freezing of Lennard-Jones (LJ) methane in slit pores, and concluded the following. Depending on the strength of the

\footnotetext{
a) Author to whom correspondence should be addressed. Electronic mail: miyahara@cheme.kyoto-u.ac.jp
}

attractive potential energy from pore walls, fluid in a slit pore in equilibrium with saturated vapor shows freezing point $\mathrm{el}$ evation as well as depression, and the critical strength to divide these two cases is the potential energy exerted by the fluid's solid state. The "excess" attraction relative to the critical one is considered to bring the confined liquid to a higher-density state that resembles a compressed state, which would result in the elevated freezing point. This result is in accord with other recent studies. Dominguez et al. ${ }^{13}$ examined freezing of LJ fluid in slit pores of purely repulsive and weakly attractive walls, employing a thermodynamic integral technique to find out true equilibrium points. The freezing points that were determined rigorously by free energy calculation showed a significant downward shift, relative to the bulk, in purely repulsive walls, while the downward shift was much smaller in magnitude for weakly attractive walls. Further, Radhakrishnan and Gubbins ${ }^{14}$ used a different approach to determine the freezing point in slit pores by employing the Landau free-energy calculation; simple fluid in strongly attractive slit pores was shown to exhibit elevated freezing points, not only theoretically but also experimentally, as reported in another paper, ${ }^{15}$ in which calorimetric measurements were conducted for carbontetrachloride confined in graphitic pores of activated carbon fiber.

Freezing of LJ fluid in pores of cylindrical geometry has been investigated by Maddox and Gubbins ${ }^{16}$ using MD simulation. Their conclusion is that the confinement in cylindrical geometry has the tendency to lower the freezing point compared with slit-shaped pores. This finding provides a qualitative understanding of freezing point depression observed in earlier experiments. As for the quantitative aspect, however, the understanding in this geometry may not be sufficient, although a trial for quantitative modeling is in progress. $^{17}$ 
Freezing phenomena in confined space must be affected, we suppose, by at least the following three factors: (i) strength of pore wall potential energy, (ii) geometrical shape of the pore, and (iii) equilibrium vapor-phase pressure. The first and second points, as explained above, have been investigated to some extent. As for the third factor, however, there does not appear to have been a systematic study, either in the theoretical or experimental aspect. In this paper, the effect of equilibrium vapor-phase pressure $p$ is examined, our motive being as follows. Contrary to the case with the saturated vapor $p_{s}$ for equilibrium bulk condition, the capillarycondensed liquid with $p / p_{s}<1$ is subjected to far lower pressure than that in the bulk. Negative pressure, or tensile conditions can easily be the case for liquids in nanopores, which should then bring a depressing effect on the freezing point. Note here that what we are aiming at is not a triple point, but the solid-liquid coexistence curve for pore space: The system is in equilibrium with vapor phase in bulk, but it does not have coexisting vapor within pore space. In other words, the pore is filled with condensed phase of either liquid or solid, because the bulk vapor pressures are, even with the condition of $p<p_{s}$, always larger than the critical condensation pressure, as illustrated later in Fig. 8.

We employ a molecular dynamics (MD) technique in a unit cell with imaginary gas phase, ${ }^{18}$ which enables us to set or obtain equilibrium vapor-phase pressure of the adsorbed phase. The MD simulations show liquid-solid phase transitions, at a constant temperature, with the variation in the equilibrium vapor-phase pressure below the saturated one. Thus-determined solid-liquid coexistence points are found to exhibit significant dependence of the freezing point onto equilibrium bulk-phase pressure, forming an extraordinarily skewed curve on a $p-T$ diagram, in contrast to the bulkphase coexistence of an almost vertical line. The origin of the significant dependence is considered to be the tensile effect in the capillary-condensed phase through examination with a simple model based on this effect.

We understand that, in nanopores, the pressure would exhibit anisotropic nature and a "pressure" cannot be treated as done in bulk phase. Nevertheless, the simple model with continuum and isotropic assumption will prove its usefulness in understanding and estimating freezing behavior in nanopores.

\section{MD SIMULATION WITH IMAGINARY GAS PHASE}

A possible method for simulating a pore fluid with its equilibrium vapor phase specified may first be the grand canonical Monte Carlo (GCMC). This method, however, is not suitable for the purpose of investigation here because of the large artificial hysteresis in condensation/evaporation encountered in GCMC. ${ }^{19}$ Suppose a reduction in equilibrium relative pressure would cause melting in a pore. We cannot, however, immediately find if the liquid state in the pore would be thermodynamically stable. It might be on a metastable branch of the condensed liquid, and the stability can be confirmed only after a complicated procedure for finding grand potential, employing a thermodynamic integral that

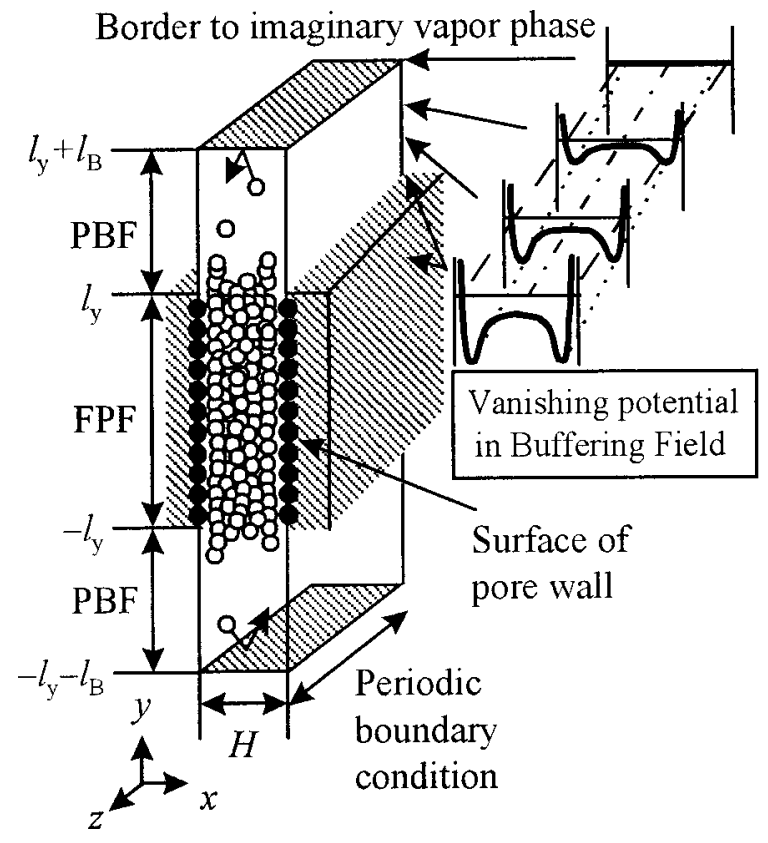

FIG. 1. Schematic figure of unit cell and potential profile within the cell. At each end of the "full potential field," a connecting space "potential buffering field" is set between the border to the imaginary gas phase. Only the $z$ direction has the periodic boundary condition.

needs a large number of simulation runs, including those at various temperatures. Instead, we employ an MD simulation scheme with an imaginary gas phase. ${ }^{18}$

\section{A. Unit cell with imaginary gas phase}

Since the details are available in Ref. 18 the feature of the simulation is only briefly explained below. Figure 1 illustrates the simulation cell. In the middle of the cell is the pore space with a given potential energy (full potential field: FPF). At each end of the cell, distant from the edge of FPF, we set a border plane beyond which an imaginary gas phase is assumed to exist. Since the external potential energy in gas phase must be zero, there should exist a connecting space with slope of potential energy between the gas phase and the pore space, which we call the potential buffering field (PBF).

The benefit of this simulation cell is reflected in the easy determination of equilibrium vapor pressure. Molecules trying to desorb from the pore space must climb up the potential slope in PBF, and only those with sufficient kinetic energy can reach the border plane. If we set a perfect reflection condition at the border, the frequency of the particles coming up should be a direct measure of the vapor pressure in bulk; that is, in equilibrium with given adsorbed phase. By simply "counting" molecules reaching the border against time, the equilibrium pressure can easily be determined (called the "particle counting method") as far as the ideal-gas assumption holds for given pressure and temperature. Note here that the FPF stays filled with the condensed phase as long as the equilibrium pressure is larger than the critical condensation pressure, though the PBF may not. Further, we found that the liquid in the cell shows almost no hysteresis in condensation/ evaporation, which is quite desirable for the purpose here. 


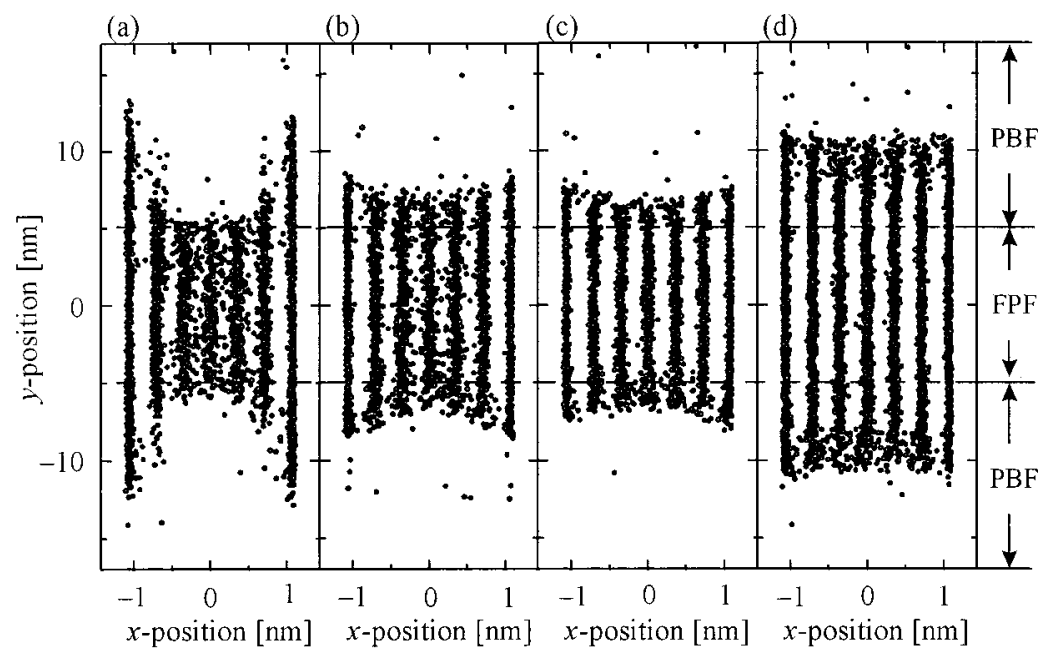

FIG. 2. Snapshots of particle positions obtained by MD simulations for a pore of $H / \sigma_{f f}=7.5$ at $T=114 \mathrm{~K}$ : (a) $p / p_{s}=0.26$, (b) $p / p_{s}=0.46$, (c) $p / p_{s}=0.49$, (d) $p / p_{s}$ $=0.89$.

\section{B. Potential functions and setting of the unit cell}

For fluid-fluid interaction, the LJ (12-6) potential

$$
u(r)=4 \varepsilon_{f f}\left[\left(\frac{\sigma_{f f}}{r}\right)^{12}-\left(\frac{\sigma_{f f}}{r}\right)^{6}\right],
$$

was used, where energy and size parameters modeled on methane $\left(\varepsilon_{f f} / k=148.1 \mathrm{~K}, \sigma_{f f}=0.381 \mathrm{~nm}\right)$ were employed. The cutoff distance of adsorbate was set to be $5 \sigma_{f f}$, which was felt to be large enough to represent particles with the full LJ potential. Thus, no long-range correction was attempted.

The pore wall was modeled on graphite. As shown in an earlier study, ${ }^{12}$ a structured wall with a potential function with lateral periodicity gave essentially the same results as those for a structureless smooth wall with the so-called 104-3 potential; only the latter was employed here

$$
\begin{aligned}
\phi_{f s}(w)= & 2 \pi \rho_{s} \varepsilon_{f s} \sigma_{f s}^{2} \Delta\left[\frac{2}{5}\left(\frac{\sigma_{f s}}{w}\right)^{10}-\left(\frac{\sigma_{f s}}{w}\right)^{4}\right. \\
& \left.-\frac{\sigma_{f s}^{4}}{3 \Delta(w+0.61 \Delta)^{3}}\right],
\end{aligned}
$$

where $w$ is a distance between a fluid particle and the center of surface atoms of the walls, and $\rho_{s}$ is the number density of carbon. The parameters $\varepsilon_{s s} / k$ and $\sigma_{s s}$ were $28.0 \mathrm{~K}$ and 0.340 $\mathrm{nm}$, respectively, and the separation between graphitic planes, $\Delta / \sigma_{s s}$, was $0.985 .^{20,21}$ The Lorentz-Berthelot combining rules were applied to obtain parameters for the methane-carbon interactions, $\varepsilon_{f s} / k$ and $\sigma_{f s}$. The potential $\psi$ at a position $x$ in a slit pore of given width $H$ was calculated as the sum of contributions from two walls: $\psi(x)$ $=\phi_{f s}(x)+\phi_{f s}(H-x)$. Other constants are the mass of the methane $m=2.665 \times 10^{-26} \mathrm{~kg}$, the number density of carbon in pore $\rho_{s}=1.14 \times 10^{29} \mathrm{~m}^{-3}$. The pore widths ranged from $5.5 \sigma_{f f}$ to $10 \sigma_{f f}$.

In PBF, the potential within FPF was attenuated linearly towards zero at the border of the imaginary gas phase. Pore fluid stays mostly in FPF under lower equilibrium pressure, but its edge comes into PBF when simulating pore fluid with higher equilibrium pressure. Maximum attention was paid to keep sufficient distance between the border plane and the edge of fluid in the pore (see, e.g., Fig. 2) so that a particle at the vapor-phase plane does not receive any interactions from the condensate in the pore: If the PBF length $l_{B}$ is insufficient, resultant equilibrium pressure suffers from overestimation because a lower number of particles, in reality, should deserve to stand for "ideal-gas particles free from fluid's potential," as examined previously. ${ }^{18}$

The existence of an interface between pore fluid and gas phase in this cell, instead of the usually employed periodic boundary condition in the $y$ direction, brings in another feature: For a condition in which pore fluid should freeze, the central portion of FPF (CFPF) holds the frozen phase, but the melted phase exists around the edge of condensate in PBF [see Figs. 2(c), 2(d)] because of a weaker potential field there and because of a somewhat parallel case with so-called "surface melting." This melted phase may, to some extent, affect the structure of solid phase in the outer portion of FPF. We should set a sufficient length of FPF to accommodate this intermediate phase in addition to CFPF: With a shorter length of FPF, the solid phase may not hold even under a condition where it should. The effect of FPF length is described in detail in Appendix A. With sufficient length of FPF, the data analysis was made for fluid particles in the CFPF, between $-5 \sigma_{f f}<y<5 \sigma_{f f}$, in order not to be affected by the melted phase near the interface.

Considering and testing the above influences (see Appendix A for FPF length), we determined the following setting. The length of the FPF, $2 l_{y}$, was $26.25 \sigma_{f f}(10 \mathrm{~nm})$ and the length of PBF, $l_{B}$, was $31.50 \sigma_{f f}(12 \mathrm{~nm})$. The size of the cell in the $z$ direction is $11.25 \sigma_{f f}$; we imposed the period boundary condition in this direction.

A simulation run for a given number of fluid particles started from an initial configuration arranged as liquid-like phase within a slit-shaped pore. The initial velocity of each particle was given so as to attain the Maxwell-Boltzmann distribution at a given temperature. The temperature of the system was controlled by velocity scaling once every 100 steps, during $1000 \mathrm{ps}$ in the beginning. The Verlet method ${ }^{22}$ was used to integrate the equations of motion numerically. Each run consisted of at least 5000 ps with time increments of $10 \mathrm{fs}$, the duration of which was decided so that the num- 
ber of particles reaching the border plane numbered about 500 or more. The total number of particles in the cell, $N_{T}$, ranged from ca. 2000 to 4000 depending on the desired condition.

\section{Consistency with GCMC results and smaller hysteresis}

The above simulation scheme with imaginary gas phase was, in an earlier paper, ${ }^{18}$ proven to give proper values of equilibrium vapor pressures in agreement with those determined by Widom's particle insertion method, ${ }^{23,24}$ as far as condensation/evaporation in a nanopore is concerned. For freezing/melting, however, the method might have different characteristics. Before exploring tensile conditions, then, the validity of the simulation method was tested, comparing the freezing/melting behavior of pore fluid under equilibrium with saturated vapor, obtained by the GCMC method. ${ }^{12}$

The details are given in Appendix B: The method is confirmed to give almost the same, and even better, results as those by the GCMC method. The meaning of "better" is in connection with hysteresis. The hysteresis in freezing/ melting inevitably occurs also in this cell, but to a much smaller extent compared with those in GCMC simulations. As noted in Ref. 12, the freezing branch in GCMC is thought to be closer to the true transition point than the melting sequence. It is thought to be true also for the simulation here. With smaller hysteresis, then, the freezing branch in our method should give a transition point closer to the true equilibrium point. This feature probably comes from the existence of interface in our cell, but exact reasoning should be withdrawn until further examination.

Here, it can be concluded that the simulation scheme employed is thought to be suitable for exploring freezing behavior in a pore under the condition of $p / p_{s}<1$, with at least a similar degree of, or even better, reliability compared with the GCMC method.

\section{RESULTS AND DISCUSSION}

In this section, variation of phase condition against relative pressure is explained, which exhibits liquid-solid phase transition at constant temperature. As explained above and in Appendix B, we are mainly interested in the freezing branch, and results observed in the sequence with increasing relative pressure are analyzed from many aspects such as snapshots, densities, diffusivities, in-plane pair correlation functions, and in-plane structure factors.

\section{A. Liquid-solid phase transition at constant $\mathbf{T}$}

Figure 2 shows typical snapshots of LJ-methane in graphite pores of $H^{*}=7.5$ at $T=114 \mathrm{~K}$ with various equilibrium vapor pressure. It might look like it has coexisting vapor in the pore. The vapor phase, however, cannot be the coexisting one in the pore, because it lies only in the PBF and because the pore space, or the FPF, is completely filled with the condensed phase. At low pressure, near the critical capillary condensation condition [Fig. 2(a)], the pore fluid exhibits liquid-like structure with a small degree of layering which is typically observed for capillary condensate in a pore

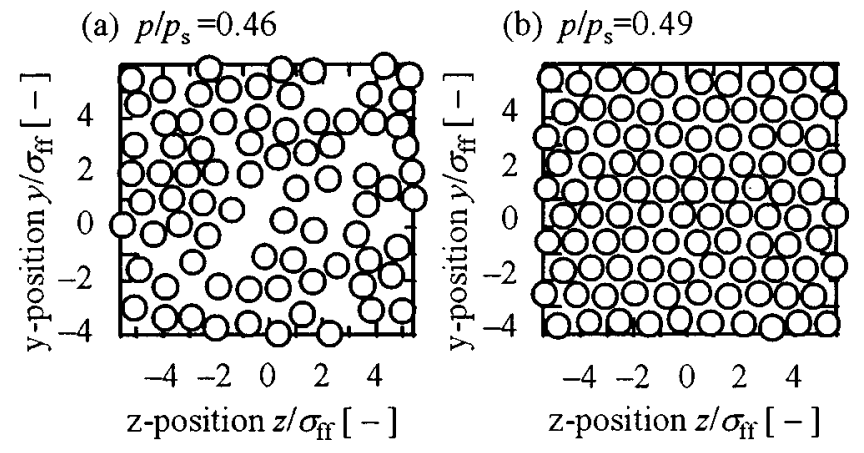

FIG. 3. Sectional snapshots for innermost layer in a pore of $H / \sigma_{f f}=7.5$ : (a) at $p / p_{s}=0.46$, showing liquid-like structure with random nature; (b) at $p / p_{s}=0.49$, exhibiting ordered structure with hexagonal array.

a few nanometers in width. Note that the $x$ scale of each figure is expanded for ease in recognition of layers. In Fig. 2(b) the degree of layering becomes more recognizable, but there some molecules still exists between the layers. Only a slight difference in relative pressure brings a solid-like state as seen in Fig. 2(c), where highly discrete distribution can clearly be recognized. Further increase in equilibrium pressure makes essentially no change in the state of molecules in the pore space (FPF: $-5 \mathrm{~nm}<y<5 \mathrm{~nm}$ ) as seen in Fig. 2(d). Under saturated vapor the system is solid-like at this temperature, and the above variation seems quite reasonable.

The above difference between Figs. 2(b) and 2(c) might look subtle, but in-plane snapshots for the innermost layer show a definite difference between the two, as shown in Fig. 3: A liquid-like structure of random nature changes to a completely ordered state with hexagonal array. It should be noted here that the contact layer adjacent to the pore wall does not participate in the change: it keeps hexagonal order before and after the change, similarly to the behavior with temperature variation observed in GCMC. ${ }^{12}$

The variation is quantitatively expressed as the overall density $\rho^{*}$ of adsorbate in the CFPF,

$$
\rho^{*}=\rho \sigma_{f f}^{3}: \rho=\frac{\langle N\rangle}{V},
$$

where $\langle N\rangle$ is the ensemble average of the number of fluid particles in the CFPF. $V$ is taken as a volume of the pore space in the CFPF between the planes of nuclei of the first layer of carbon atoms of the walls, which includes some dead space in the vicinity of the walls where particle centers essentially cannot penetrate. By this definition the overall density is smaller than the true density of pore fluid. As shown in Fig. 4, the density exhibits almost vertical change around $p / p_{s}=0.48$. In the range of smaller relative pressure, the density shows a gradual increase against pressure, indicating liquid-like structure, while in the higher range almost no variation in density can be recognized.

We calculated the self-diffusion coefficient of the particles in the CFPF. Molecular dynamics technique in general benefits by this dynamic property, with which characteristics of a phase can more clearly be decided. The self-diffusion 


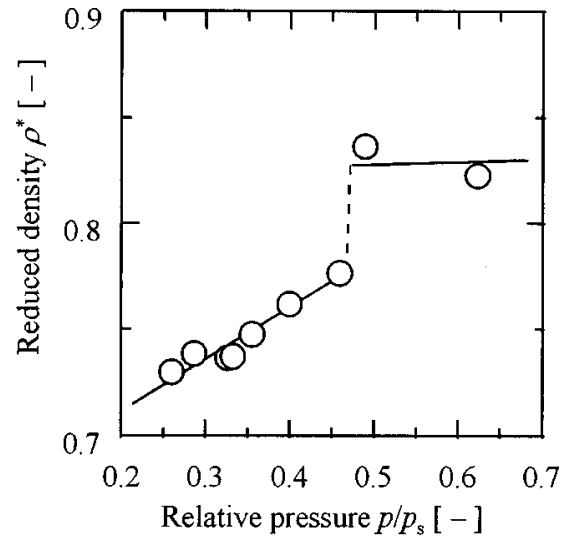

FIG. 4. Variation of overall density within a pore of $H / \sigma_{f f}=7.5$ at $T=114$ $\mathrm{K}$ against equilibrium vapor-phase pressure. Discrete change in density corresponds to a liquid-like to solid-like transition.

coefficients in the $y$ direction $D_{y}$ were obtained from the mean-square displacements in the direction with the following equation: ${ }^{25}$

$$
D_{y}=\lim _{t_{k} \rightarrow \infty} \frac{1}{2 t_{k}}\left\langle\Delta y^{2}\left(t_{k}\right)\right\rangle,
$$

where

$$
\left\langle\Delta y^{2}\left(t_{k}\right)\right\rangle=\frac{1}{N(t)} \sum_{i}^{N}\left[y_{i}\left(t_{i}\right)-y_{i}\left(t_{i}+t_{k}\right)\right]^{2},
$$

$N$ is the number of particles in the CFPF. The reduced selfdiffusion coefficient $D^{*}$ is $D /\left(\varepsilon_{f f} \sigma_{f f}^{2} / m\right)^{1 / 2}$. When a particle goes out of the CFPF space, the trace of the particle is finished. If a particle comes into the CFPF, we add 1 to $N$ and begin to trace the movement of the coming particle with the initial time for this particle $t_{i}$. In this analysis, the summation of the mean-square displacement of each particle with a different time origin is used in Eq. (4). Thus, the number of particles was not constant but a function of time. $D_{y}$ was decided from slope of the average mean-square displacement against $t_{k}$, an example of which is illustrated in Fig. 5.

Figure 6 shows dependency of the diffusivity, which exhibits a rather steep decrease against relative pressure in the

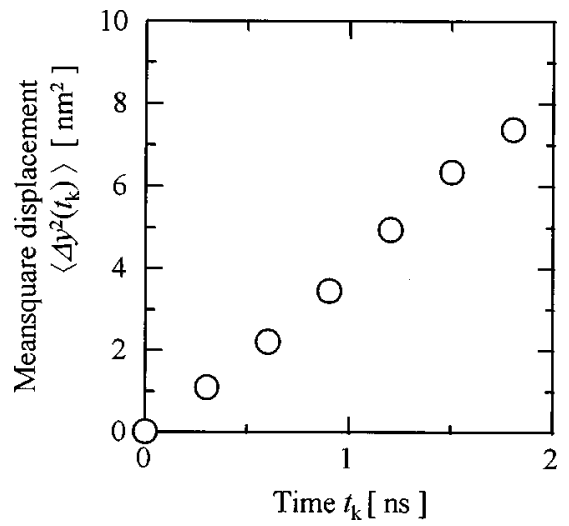

FIG. 5. Example of mean-square displacement plot for determination of self-diffusivity: $H / \sigma_{f f}=7.5, T=114 \mathrm{~K}$, and $p / p_{s}=0.35$. The slope gives self-diffusivity.

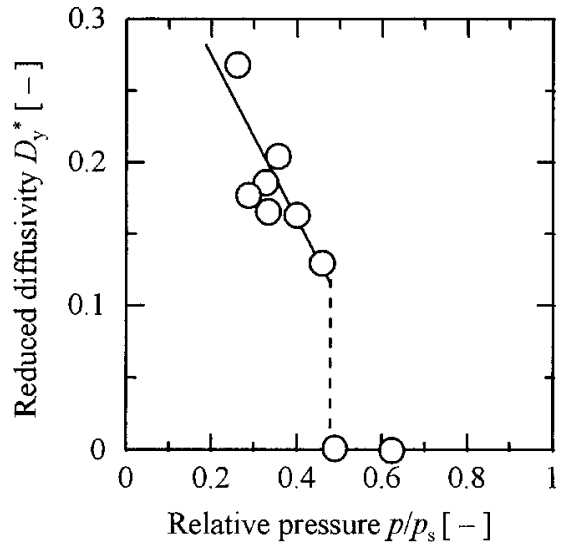

FIG. 6. Calculated self-diffusivity in the $y$ direction plotted against equilibrium pressure. At the same relative pressure as that for the step change in density in Fig. 5, diffusivity drops to be negligibly small, indicating transition to a solid-like phase.

lower range, and nearly vanishes to be negligibly small above $p / p_{s}=$ ca. 0.48: in the higher range of $p / p_{s}$ each particle in the phase essentially stays at a position, and does not move within the simulation time. This observation clearly demonstrates that the change around $p / p_{s}=0.48$ is associated with liquid-solid phase transition.

Further, though not shown here, we calculated in-plane pair correlation functions and static structure factors for individual layers, and all the results support that the step-like change against relative pressure is a transition from a disordered liquid-like state to an ordered solid-like state: These functions showed a quite similar manner of change to those observed with temperature variation in GCMC simulations. ${ }^{12}$ Since the critical condensation condition for this pore is certainly lower than this transition point to stay around $p / p_{s}$ =ca. 0.2 , the liquid-like state is not on a metastable branch, but thermodynamically stable. Thus, a solid-liquid coexistence point is determined for this temperature. It may have a slight overestimation in relative pressure, but it can stand for a coexistence point as a good approximation because of the nature of the freezing branch and because of the small degree of hysteresis, as explained in Sec. II C.

\section{B. Liquid-solid coexistence curves below saturated vapor pressure}

The above set of simulations was conducted for various temperatures, and the coexistence points were determined as shown in Fig. 7 along with the bulk coexistence lines for this LJ fluid. ${ }^{26,27}$ Also, a similar series of simulations was conducted for other sizes of pores ranging from $5.5 \sigma_{f f}$ to $10 \sigma_{f f}$, and plotted in the figure. What is most striking here would be the quite significant dependence of the freezing point against the bulk-phase pressure below the saturated vapor line of bulk fluid. Normal bulk fluid shows only a weak change in freezing point against pressure, as described by the almost vertical solid-liquid coexistence. Thus, the observed depression in freezing below the saturated vapor line cannot be explained simply by the mechanical effect of pressure varia- 

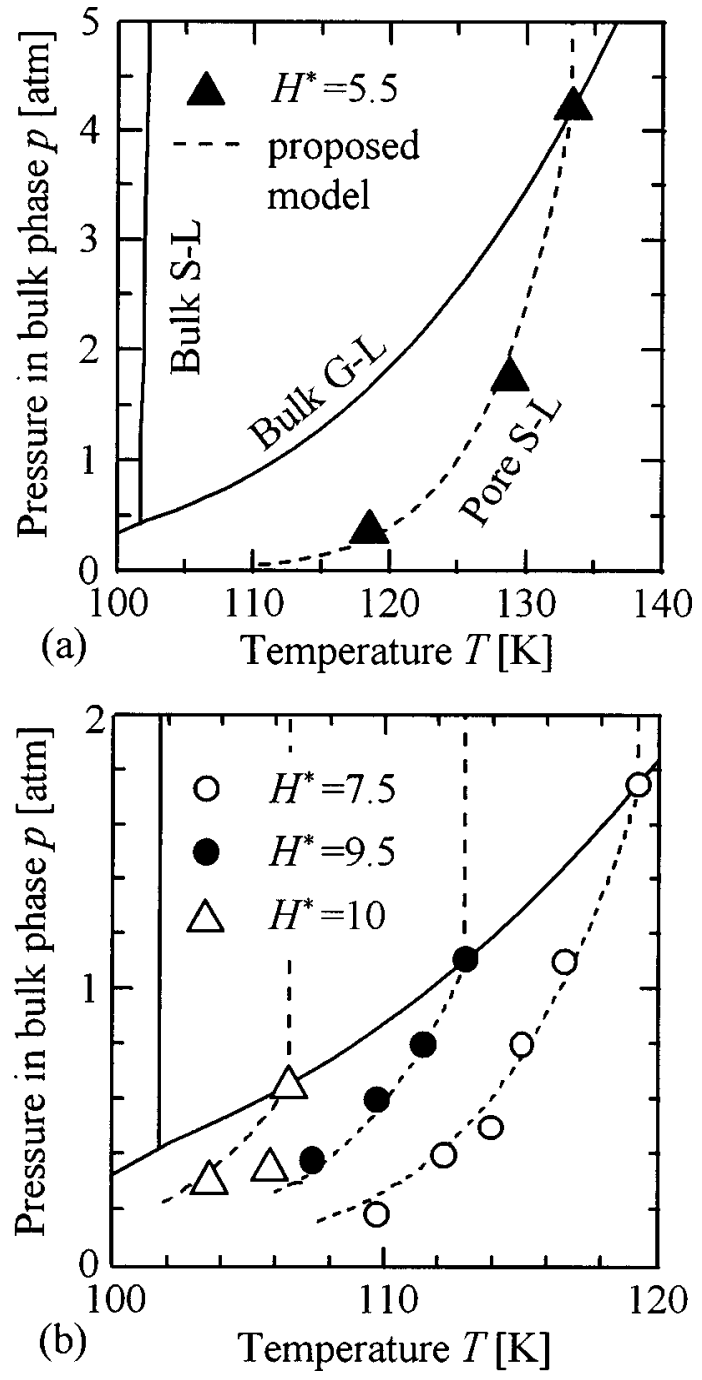

FIG. 7. Liquid-solid coexistence curves for pore fluid in various pore widths obtained with MD simulations (keys), superimposed on bulk-phase diagram (solid lines): (a) for $H / \sigma_{f f}=5.5$; (b) for $H / \sigma_{f f}=7.5,9.5$, and 10 . Dashed lines are predictions given by the simple model.

tion in bulk. Such degree of freezing depression, however, is thought to be corresponding to significant variation of the pressure felt by the capillary fluid.

This presumption about the pressure may be supported qualitatively by the snapshots shown in Fig. 2. The interface exhibits strong curvature when the pore phase is liquid-like, while it becomes rather flat with solid-like phase under high relative pressure: a strong Young-Laplace effect is thought to be prevailing to hold the melted phase with lower relative pressures. The observation in Fig. 7 that stronger dependence arises for smaller pores would be further support for the importance of the capillary effect. Quantitative analysis of the shape of the interface, as done by the authors for capillary condensation, ${ }^{28}$ however, cannot be made here because it holds in the attenuating potential field, PBF, in which not only the $x$ dependent but also the $y$-dependent potential field affects the treatment of the shape. Also, even in the full potential field, strict treatment of pressure in the pore phase would be very complicated because the potential field brings significant direction dependence in the pressure tensor. We

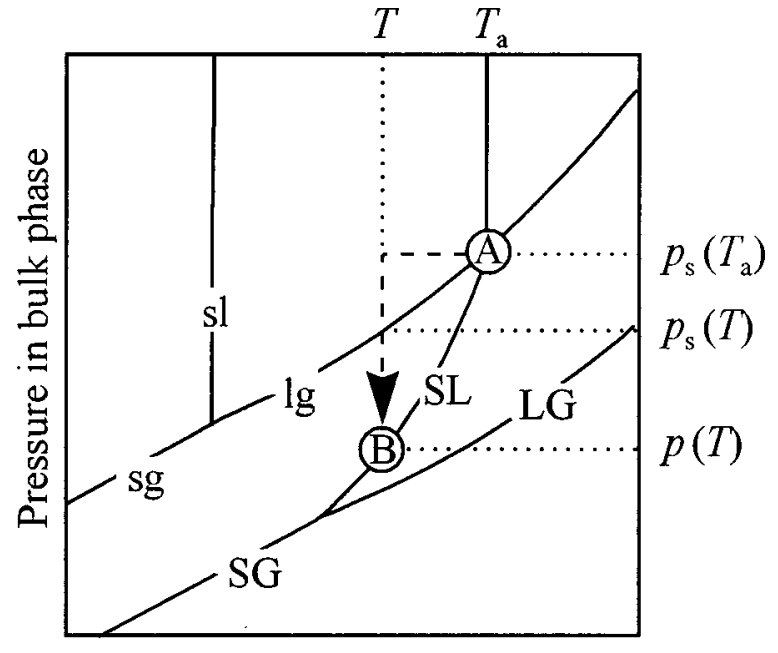

Temperature

FIG. 8. Schematic phase diagram for model consideration. Point A indicates a freezing point of pore fluid in equilibrium with saturated vapor. Point $B$ stands for a coexistence point on the phase boundary of pore fluid under the tensile condition.

do not try this kind of strict treatment here. Instead, in the next section, we try a most simple treatment.

\section{Simple model}

Here, we examine to what extent the simple concept of continuum media with isotropic pressure can model the freezing phenomena observed above. Again, we understand that this is not scientifically correct in nanopores, but our aim is to seek a simple model for understanding and estimating the freezing points in pores.

As a starting point, we take freezing point under saturated vapor, point $\mathrm{A}$ in Fig. 8, in which phase coexistence curves for bulk fluid (thin lines) and pore fluid (thick lines) are schematically illustrated. As depicted in the figure, freezing point elevation is the case for slit pores with strongly attractive potential. The degree of the elevation $\delta T$, relative to bulk freezing point $T_{f}$, was modeled previously to be $\mathrm{e}^{12}$

$$
\frac{\delta T}{T_{f}}=-\frac{\Delta \psi}{\Delta h_{m}},
$$

where $\Delta h_{m}=\left(s_{L}-s_{S}\right) T_{f}$ is the latent heat absorbed on melting, and is positive. $\Delta \psi\left(=\psi-\psi^{\text {methane }}\right)$ is the excess amount of potential energy in the pore relative to the one from fictitious pore walls made up of the fluid's solid state, and is negative for strongly attractive walls. This equation was shown to give freezing points in fairly good agreement with those observed in molecular simulations.

Suppose we have a solid-liquid coexistence point $(T, p)$ for pore fluid on the bulk phase diagram as shown by point B in Fig. 8. Though the bulk pressure is at $p$, the fluid in the pore is supposed to have different pressure $p^{\text {pore }}$ because of the pore-wall potential and the capillary effect. Not for the bulk pressure but for this pressure felt by fluid in the pore, $p^{\text {pore }}$, is the Clausius-Clapeyron equation for the bulk assumed to hold. 


$$
\frac{d p^{\text {pore }}}{d T}=\left(\frac{\Delta s}{\Delta v}\right)_{\text {pore }} \cong\left(\frac{\Delta s}{\Delta v}\right)_{\text {bulk }} \cong \text { const. }
$$

To find the freezing point shift relative to the freezing temperature $T_{a}$ at $p=p_{s}$, the difference in $p^{\text {pore }}$ between the points $\mathrm{A}$ and $\mathrm{B}$ is considered below, in which physical properties of pore fluid are approximated by those for bulk, which is thought to be appropriate for slit pores because solid phase in pore forms face-centered cubic (fcc) structures resembling those for bulk phase: for other geometry, e.g., cylindrical, this assumption may fail to hold.

For bulk phase, the chemical potential difference $\Delta \mu^{\text {bulk }}$ between $\mathrm{A}$ and $\mathrm{B}$ can be expressed as follows, considering the path along the broken line in the figure:

$$
\begin{aligned}
\Delta \mu^{\text {bulk }} & =\mu^{\text {bulk }}(T, p)-\mu^{\text {bulk }}\left(T_{a}, p_{a}\right) \\
& =-\int_{T_{a}}^{T} s_{L} d T+\int_{p_{a}}^{p_{s}(T)} v_{L} d p+k T \ln \frac{p}{p_{s}(T)} \\
& \cong-s_{L}\left(T-T_{a}\right)+k T \ln \frac{p}{p_{s}(T)} .
\end{aligned}
$$

In the above, the entropy and molar volume of bulk liquid, $s_{L}$ and $v_{L}$, are assumed to be constant within the range considered, and the ideal gas law is applied for vapor phase. The $v_{L} d p$ term is neglected, compared to the first and the third term. On the other hand, tracing the pore SL coexistence, the difference in chemical potential for pore liquid $\Delta \mu^{\text {pore }}$ between $\mathrm{A}$ and $\mathrm{B}$ can be approximately written as

$$
\begin{aligned}
\Delta \mu_{L}^{\text {pore }} & =-\int_{T_{a}}^{T} s_{L}^{\text {pore }} d T+\int_{\left.p^{\text {pore }}\right|_{A}}^{p^{\text {pore }} v_{B}} v_{L}^{\text {pore }} d p^{\text {pore }} \\
& \approx-s_{L}\left(T-T_{a}\right)+v_{L}\left(\left.p^{\text {pore }}\right|_{B}-\left.p^{\text {pore }}\right|_{A}\right) .
\end{aligned}
$$

Equating the above two, we can obtain the difference in $p^{\text {pore }}$ as follows:

$$
\left.p^{\text {pore }}\right|_{B}-\left.p^{\text {pore }}\right|_{A}=\Delta p^{\text {pore }} \cong \frac{k T}{v_{L}} \ln \frac{p}{p_{S}(T)} .
$$

The above equation is intuitively understandable because it is the basis for the Kelvin equation: If the pressure difference is equated with the Young-Laplace equation, it yields the Kelvin one. It should be noted here that Eq. (9) does not suffer from the incorrectness of the Kelvin equation for nanopores because it does not include any pore-size-related factors.

Thus, knowing the pressure difference for pore fluid, integration of Eq. (6) and rearrangement will yield the following equation to describe the relation between freezing point of the tensile fluid in pore and the bulk-phase pressure on the bulk phase diagram:

$$
p=p_{s}(T) \exp \left[-\left(\frac{\Delta s}{\Delta v}\right)_{\text {bulk }} \frac{v_{L}}{k T}\left(T_{a}-T\right)\right] .
$$

We examined the performance of this simple model by comparing it with the simulation results. For LJ fluid, bulk properties such as $v_{L}$, and $\Delta s / \Delta \mathrm{v}$ are well known. ${ }^{26,27} T_{a}$, the freezing point under saturated vapor, can be estimated by Eq. (5). Thus, the above equation includes no adjustable pa- rameters. The dashed lines in Fig. 7 are the calculated results of Eq. (10) for each pore size. Surprisingly, such a simple model gives quite a good performance in expressing freezing point shift under tensile condition in a pore of width as small as $2-4 \mathrm{~nm}$. This agreement demonstrates usefulness of the concept of the effective pressure felt by the pore fluid in understanding the freezing in nanopores.

If combining this simple model with a model for capillary condensation proposed by the authors, ${ }^{17,28}$ triple points in nanopores are thought to be predictable. Molecular simulations for finding triple points in a nanopore, followed by examination of the model, would clarify the possibility, which will soon be reported. ${ }^{29}$

\section{CONCLUSION}

The effect of equilibrium vapor-phase pressure on freezing in confined space was examined employing a molecular dynamics (MD) technique in a unit cell with imaginary gas phase. The MD simulations showed liquid-solid phase transitions, at a constant temperature, with the variation in the equilibrium vapor-phase pressure below the saturated one. Thus-determined solid-liquid coexistence lines exhibited significant dependence of the freezing point against a small change in the bulk-phase vapor pressure, which implies importance of the tensile effect on freezing in nanopores.

A simple model to describe the lowering of the freezing point was derived in terms of the capillary effect that would reduce an effective pressure felt by the pore fluid, and showed good agreement with the simulation results. In nanopores, pressure exhibits anisotropic nature and a "pressure" cannot be treated as in the bulk phase. Nevertheless, the viewpoint of "the pressure felt by the confined fluid" showed its usefulness in understanding and estimating freezing behavior in nanopores. Since in slit pores the structure of solid phase shows a similar nature to that in bulk, the model is able to stand with bulk properties of simple fluid and needs no adjustable parameter. For a more complicated geometry, one may need to include some unknown parameter related to the structure of pore solid in that geometry.

\section{ACKNOWLEDGMENTS}

It is a pleasure to thank Keith E. Gubbins for helpful discussions. This work was supported in part by the Grantin-Aid for Scientific Research, No. 10555266, provided by the Ministry of Education of Japan.

\section{APPENDIX A: EFFECT OF FPF LENGTH}

In addition to the results of Fig. 4 with FPF length of 10 $\mathrm{nm}\left(26.25 \sigma_{f f}\right)$, which is the final setting, simulations with two other length of $2 l_{y}, 4$ and $2 \mathrm{~nm}$, have been conducted under various conditions. Figure 9 shows an example of results expressed as variation of density against equilibrium pressure for $H / \sigma_{f f}=7.5$ at $114 \mathrm{~K}$. Obviously, $2 \mathrm{~nm}$ $\left(=5.25 \sigma_{f f}\right)$ is too short to hold the solid phase: Even under high relative pressure, the pore fluid cannot get a higher density than ca. 0.75 . On the other hand, the other two series with longer FPF exhibit liquid-solid transitions, and they give essentially the same results. Thus, even $4 \mathrm{~nm}$ 


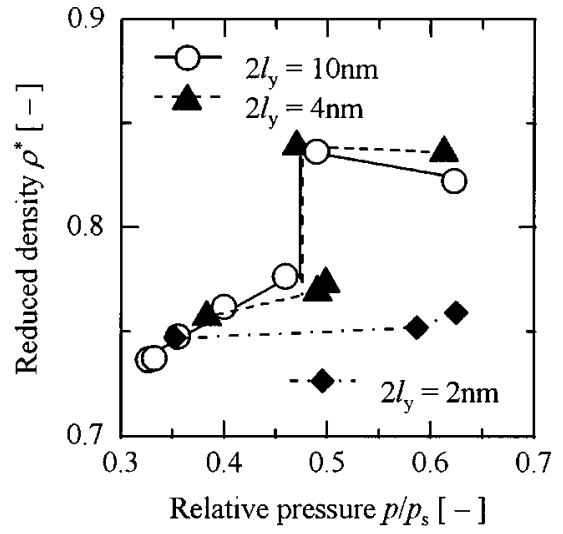

FIG. 9. Effect of FPF length on behavior of pore fluid in $H / \sigma_{f f}=7.5$ at $T=114 \mathrm{~K}$.

$\left(10.50 \sigma_{f f}\right)$ is thought to be sufficient to examine the fluid's behavior. To make doubly sure, the longer one $(10 \mathrm{~nm})$ is finally employed as a standard condition for the simulations.

\section{APPENDIX B: HYSTERESIS LOOP IN MELTING/ FREEZING AND COMPARISON WITH GCMC RESULTS}

The simulation scheme with imaginary gas phase was tested comparing with freezing/melting behavior under equilibrium with saturated vapor, obtained by the GCMC method. ${ }^{12}$ The same sequence as that employed in Ref. 12 was conducted with the MD simulations: Cooling and heating sequences along the bulk GL coexistence line were followed. Since the equilibrium vapor phase pressure in our MD simulation is NOT an input parameter but an output one, slight deviation from saturated vapor pressure was sometimes the case. Only those results with deviation less than $5 \%$ were employed for the test.

Results with pore width $H / \sigma_{f f}=5.5$ were illustrated in Fig. 10, which shows almost the same behavior as that ob-

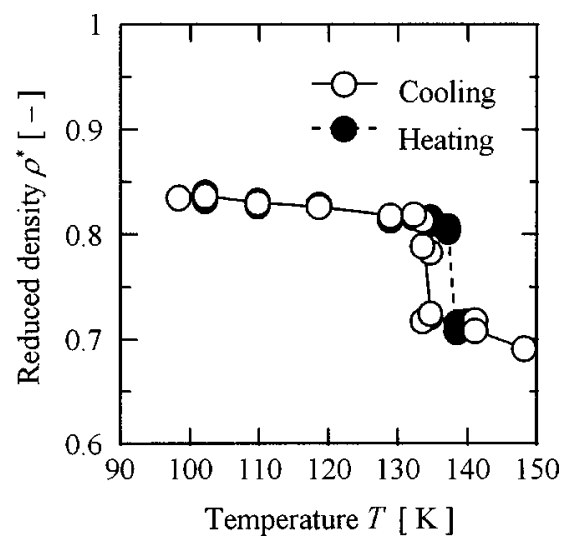

FIG. 10. Variation of fluid's density in a pore of $H / \sigma_{f f}=5.5$ obtained by MD method following sequence with varying temperature: open circles, cooling; closed circles, heating. Hysteresis is less pronounced than GCMC, with almost the same freezing temperature. served in the GCMC simulations reported in Ref. 12. Further, GCMC gave a freezing temperature of $132 \mathrm{~K}$, and melting of $143 \mathrm{~K}$, showing rather pronounced hysteresis for this pore size, while our MD results are 133 and $137 \mathrm{~K}$, respectively, with a much smaller degree of hysteresis with almost the same temperature in the freezing branch as that in GCMC. This observation confirms consistency of the employed MD simulation with the GCMC method, and demonstrates an even better feature of it.

As for the sequence in which equilibrium pressure varies at constant temperature, appropriateness of taking the freezing branch, or the branch with increasing vapor pressure, was suggested from the following result. Knowing the GCMC method gave a freezing temperature of $117 \mathrm{~K}$ in a pore of $7.5 \sigma_{f f}$ under saturated vapor, we examined both branches of increasing and decreasing pressure. The increasing branch was found to exhibit transition to solid at an equilibrium pressure close to the saturated one: The branch of increasing vapor pressure, thus, corresponds to the freezing branch in temperature-variation sequence, which is thought to be closer to the true transition point than the melting sequence.

${ }^{1}$ W. A. Patrik and W. A. Kemper, J. Chem. Phys. 42, 369 (1938).

${ }^{2}$ G. K. Rennie and J. Clifford, J. Chem. Soc., Faraday Trans. 1 73, 680 (1977).

${ }^{3}$ J. L. Tell and H. J. Maris, Phys. Rev. B 28, 5122 (1983).

${ }^{4}$ J. Warnock, D. D. Awschalom, and M. W. Shafer, Phys. Rev. Lett. 57, 1753 (1986).

${ }^{5}$ R. H. Torii, H. J. Maris, and G. M. Seidel, Phys. Rev. B 41, 7167 (1990).

${ }^{6}$ P. E. Sokol, W. J. Ma, K. W. Herwig, W. M. Snow, Y. Wang, J. Koplik, and J. R. Banavar, Appl. Phys. Lett. 61, 777 (1992).

${ }^{7}$ J. H. Strange, M. Rahman, and E. G. Smith, Phys. Rev. Lett. 71, 3589 (1993).

${ }^{8}$ K. M. Unruh, T. E. Huber, and C. A. Huber, Phys. Rev. B 48, 9021 (1993).

${ }^{9}$ E. Moltz, A. P. Y. Wong, M. H. W. Chan, and J. R. Beamish, Phys. Rev. B 48, 5741 (1993).

${ }^{10}$ J. Klein and E. Kumacheva, Science 269, 816 (1995).

${ }^{11}$ J. A. Duffy, N. J. Wilkinson, H. M. Fretwell, M. A. Alam, and R. Evans, J. Phys.: Condens. Matter 7, L713 (1995).

${ }^{12}$ M. Miyahara and K. E. Gubbins, J. Chem. Phys. 106, 2865 (1997).

${ }^{13}$ H. Dominguez, M. P. Allen, and R. Evans, Mol. Phys. 96, 209 (1999).

${ }^{14}$ R. Radhakrishnan and K. E. Gubbins, Mol. Phys. 96, 1249 (1999).

${ }^{15}$ R. Radhakrishnan, K. E. Gubbins, A. Watanabe, and K. Kaneko, J. Chem. Phys. 111, 9058 (1999).

${ }^{16}$ M. W. Maddox and K. E. Gubbins, J. Chem. Phys. 107, 9659 (1997).

${ }^{17}$ H. Kanda, M. Miyahara, and K. Higashitani, Langmuir (submitted).

${ }^{18}$ M. Miyahara, T. Yoshioka, and M. Okazaki, J. Chem. Phys. 106, 8124 (1997).

${ }^{19}$ See, e.g., S. Jiang, C. L. Rhykerd, and K. E. Gubbins, Mol. Phys. 79, 373 (1993).

${ }^{20}$ W. A. Steele, Surf. Sci. 36, 317 (1973).

${ }^{21}$ W. A. Steele, The Interaction of Gases with Solid Surface (Pergamon, Oxford, 1974).

${ }^{22}$ L. Verlet, Phys. Rev. 159, 98 (1967).

${ }^{23}$ B. Widom, J. Chem. Phys. 39, 2808 (1963).

${ }^{24}$ B. Widom, J. Stat. Phys. 19, 563 (1978).

${ }^{25}$ See, e.g., M. Schoen, J. Chem. Phys. 88, 1394 (1988).

${ }^{26}$ D. A. Kofke, J. Chem. Phys. 98, 4149 (1993).

${ }^{27}$ R. Agrawal and D. A. Kofke, Mol. Phys. 85, 43 (1995).

${ }^{28}$ M. Miyahara, H. Kanda, T. Yoshioka, and M. Okazaki, Langmuir (in press); T. Yoshioka, M. Miyahara, and M. Okazaki, J. Chem. Eng. Jpn. 30, 274 (1997).

${ }^{29}$ H. Kanda, M. Miyahara, and K. Higashitani (unpublished). 